\title{
Genes associated with inflammation and the cell cycle may serve as biomarkers for the diagnosis and prognosis of acute myocardial infarction in a Chinese population
}

\author{
JIANG SU, CHANGQING GAO, RONG WANG, CANGSONG XIAO and MING YANG \\ Department of Cardiovascular Surgery, Chinese PLA General Hospital, Beijing 100039, P.R. China
}

Received October 24, 2017; Accepted April 3, 2018

DOI: $10.3892 / \mathrm{mmr} .2018 .9077$

\begin{abstract}
The present study aimed to identify biomarkers for the clinical diagnosis of acute myocardial infarction (AMI) in a Chinese population using microarray data collected from the Gene Expression Omnibus database under accession number GSE97320. This included the peripheral blood samples of three patients with AMI and three controls. Differentially expressed genes (DEGs) were identified using the limma package and protein-protein interaction networks were constructed using data from the Search Tool for the Retrieval of Interacting Genes database, followed by module analysis to screen for hub genes. Functional enrichment analyses were performed using the Database for Annotation, Visualization and Integrated Discovery. The identified genes were verified by overlapping with the target genes of microRNAs (miRs) known to be associated with AMI, as well as the DEGs identified in other AMI datasets, including GSE24519, GSE34198 and GSE48060. As a result, 752 DEGs (449 upregulated and 303 downregulated) were identified in the GSE97320 dataset. The upregulated DEGs were predicted to participate in inflammatory pathways, including the toll-like receptor (TLR) signaling pathway, including ras-related $\mathrm{C} 3$ botulinum toxin substrate 1 (RAC1), TLR4, C-C motif chemokine receptor (CCR)1; cytokine-cytokine receptor interaction, including signal transducer and activator of transcription (STAT)3; chemokine signaling pathway, including CCR10; pathways associated with cancer, including colony stimulating factor 3 receptor (CSF3R); and leukocyte transendothelial migration, including matrix metallopeptidase 9 (MMP9). The downregulated DEGs were associated with the cell cycle, including alstrom syndrome protein 1 (ALMS1). These conclusions were made following functional analysis of the genes in the three identified modules. MMP9, TLR4, STAT3, CCR1 and ALMS1
\end{abstract}

Correspondence to: Dr Changqing Gao, Department of Cardiovascular Surgery, Chinese PLA General Hospital, 28 Fuxing Road, Haidian, Beijing 100039, P.R. China

E-mail: gaochangqing_gcq@sina.com

Key words: acute myocardial infarction, biomarkers, diagnosis, microRNA, inflammation, cell cycle were regulated by miR-21-5p, whereas RAC1 was regulated by miR-30c-5p. A comparison among the four datasets confirmed the roles of CSF3R and CCR10. HtrA serine peptidase 1 (HTRA1) was the only gene associated with both mortality and recurrence. In conclusion, inflammation-associated genes, including STAT3, CCR1, RAC1, MMP9, CCR10, CSF3R and HTRA1, as well as cell cycle-associated genes such as ALMS1, may be biomarkers for the diagnosis and prognosis of AMI in Chinese people.

\section{Introduction}

Acute myocardial infarction (AMI) is the most common and serious cardiovascular disease that can induce rapid heart failure and sudden cardiac mortality. Although a declining trend of AMI has been observed due to economic development, its incidence remains high at approximately 44.57 per 100,000 individuals in 2013 (1). In addition, the mortality rate in China was estimated to increase from 1987 to 2014 by 5.6-fold (2), particularly amongst young females (3). Thus, the development of strategies for the early diagnosis of AMI to prevent the occurrence of sudden mortality is increasingly important.

Other than ischemic symptoms, physical examination and electrocardiogram changes, cardiac troponins are the most commonly used biomarker for the clinical diagnosis of AMI $(4,5)$. However, their sensitivity and specificity may be limited, as increased cardiac troponin levels are also observed in patients with non-acute coronary syndrome (6-8). Therefore, research has attempted to develop more sensitive and specific or combined type biomarkers for AMI. Mcmahon et al (9) evaluated the diagnostic efficacy of heart-type fatty acid-binding protein (H-FABP), cardiac troponin I (cTnI), creatine kinase-MB and myoglobin for the early detection of AMI and demonstrated that H-FABP has the greatest sensitivity at 0-3 h $(64.3 \%)$ and 3-6 h $(85.3 \%)$ following the onset of chest pain. The combination of cTnI and H-FABP measurements increases the levels of sensitivity to $71.4,88.2$, and $92.4 \%$ at 0-3, 3-6 and 6-12 h, respectively (9). Similarly, Pyati et al (10) indicated that H-FABP has additional diagnostic power for the early diagnosis of AMI, with its diagnostic efficiency markedly higher than that of CK-MB and myoglobin within the first $6 \mathrm{~h}$ of chest pain (10). Wang et al (11) used receiver operating characteristic curve analysis to reveal that the diagnostic accuracy of circulating monomeric C-reactive protein may be as 
high as 0.928 [95\% confidence interval (CI), 0.887-0.969] for AMI (11), whereas Han et al (12) suggested that the plasma level of RING finger protein 207 may detect individuals with AMI with $90.5 \%$ sensitivity and $100 \%$ specificity (12). However, a limited number of genes were associated with the occurrence of AMI.

The aim of the current study was to further identify new biomarkers for the clinical diagnosis of AMI via an analysis of the gene-expression differences of peripheral blood samples between Chinese patients with AMI and healthy controls.

\section{Materials and methods}

AMI microarray data. Transcriptomics data were collected from the National Center of Biotechnology Information Gene Expression Omnibus database (NCBI GEO; www.ncbi.nlm. nih.gov/geo) under accession number GSE97320 (study not published). This database contained the peripheral blood samples of three patients with AMI (mean age, 53.0 13.1 ) and three healthy controls (mean age, 53.7 \pm 4.7 ). These participants were Han Chinese and were recruited from the Department of Cardiology at China-Japan Union Hospital, Jilin University of China (Changchun, China).

Data normalization and differentially expressed gene (DEG) identification. Raw Affymetrix Human Gene CEL files were preprocessed and normalized using the Robust Multichip Average algorithm (13) using the Bioconductor affy package (version 1.34.2; www.bioconductor. org/packages/release/bioc/html/affy.html) in R (version 3.4.1; http://www.R-project.org/) (14). The DEGs between patients with AMI and healthy controls were screened using the limma method (15) using the Bioconductor package in R (version 3.32.5; www.bioconductor.org/packages/release/bioc/html/limma.html). $\mathrm{P}<0.05$ and $\log$ fold change $(\mathrm{FC}) \mid>1$ were set as the threshold values. A heatmap of the top 50 DEGs was constructed using the pheatmap package in $\mathrm{R}$ (Version 0.7.7; cran.r-project. org/web/packages/pheatmap/index.html).

Protein-protein interaction (PPI) network construction. To screen for the crucial genes associated with AMI, DEGs were mapped into the PPI data retrieved from the Search Tool for the Retrieval of Interacting Genes database (version 10.0; www.string-db.org) (16) to construct the PPI network; the cut-off value was set as a combined score of $>0.9$. The PPI network was visualized using Cytoscape 2.8 (www.cytoscape. org) (17). The hub genes with the highest degree were selected and plotted using the ggplot2 package in $\mathrm{R}$ (version 1.0.0; cran.r-project.org/web/packages/ggplot2/index.html). To identify functionally related and highly interconnected clusters within the PPI network, a module analysis was subsequently performed using the Molecular Complex Detection (MCODE) plugin of Cytoscape with the following parameters: Degree cutoff, 6 ; node score cutoff, 0.2 ; k-core, 5; and maximum depth, 100 (version 2.2; www.baderlab.org/Software/MCODE) (18). Modules with MCODE scores of $\geq 5$ and $\geq 6$ nodes were considered significant.

microRNA (miRNA/miR) prediction. To further screen for the crucial genes associated with AMI, microRNAs known to be associated with AMI were obtained from the mir2disease database (www.mir2disease.org) that documents 1,939 relationships between 349 human microRNAs and 163 human diseases based on a review of $>3,273$ published papers (19). The target genes of the AMI-associated miRNAs were predicted using the miRWalk2 database (zmf.umm. uni-heidelberg.de/apps/zmf/mirwalk2) (20), which provides the largest collection of predicted and experimentally verified miR-target interactions using various miRNA databases. The predicted target genes were subsequently overlapped with the DEGs to obtain the miRNA-DEGs interaction relationships, which were used to construct the miRNA-DEG interaction network. These genes were visualized using Cytoscape 2.8 (www.cytoscape.org) (17).

Functional enrichment analysis. Kyoto Encyclopedia of Genes and genomes (KEGG) (21) pathway and Gene Ontology (GO) (22) enrichment analyses were performed to explore the underlying functions of all DEGs, genes in modules and miRNA-DEG networks using the Database for Annotation, Visualization and Integrated Discovery (DAVID) online tool (version 6.8; david.abcc.ncifcrf.gov). $\mathrm{P}<0.05$ was selected as the threshold for determining the significant enrichment for GO and KEGG analyses.

Validation analysis. AMI microarray data were also collected from other countries via the NCBI GEO database under accession number GSE24519 (study not published). This database includes the whole blood samples of 17 Italian patients with AMI and 2 healthy individuals. Two biological repeats were performed for each patient, resulting in 34 AMI and four control samples for analysis.

To investigate the relationship between the DEGs under investigation and various clinical characteristics, the microarray datasets of GSE34198 (23) and GSE48060 (24) were also downloaded from the NCBI GEO database. In the GSE34198 dataset (23), 97 peripheral whole blood samples were obtained, including 45 from 41 patients with AMI (four patients had two repeats) who were alive during the follow-up period, four from four patients with AMI who succumbed to the disease during the 6 month follow-up period, and 48 from 45 controls (three patients had two repeats) from the Czech Republic. In the GSE48060 dataset (24), 52 peripheral whole blood samples were available, including 21 controls and patients with AMI from the USA with $(n=5)$ or without $(n=22)$ any recurrent events over an 18-month follow-up period.

All of the validated datasets were preprocessed and the DEGs between the AMI and control groups as well as between the subgroups of patients with AMI who succumbed to the disease (recurrence) and those who lived (non-recurrence) were identified using the methods similar to those described above for the GSE97320 dataset. To visualize the shared DEGs between the validated datasets and the Chinese dataset GSE97320, a Venn diagram was generated using a web-based tool (bioinformatics.psb.ugent.be/webtools/Venn).

\section{Results}

Identification of the DEGs in GSE97320. Following data normalization, 752 genes were identified as DEGs between 
the patients with AMI and healthy controls according to the thresholds of $\mathrm{P}<0.05$ and $\mid \log \mathrm{FCl}>1$, including 449 upregulated and 303 downregulated genes (Table I). Heatmap clustering analysis indicated that the identified DEGs easily distinguished patients with AMI from healthy controls (Fig. 1).

Functional enrichment analysis of DEGs. The potential functions of the DEGs were predicted using the online tool DAVID. It was demonstrated that $28 \mathrm{KEGG}$ pathways were enriched in the upregulated DEGs, such as the nuclear factor (NF)- $\kappa \mathrm{B}$ signaling pathway, including toll-like receptor (TLR)4; the TLR signaling pathway, including ras-related C3 botulinum toxin substrate 1 (RAC1), TLR4 and C-C motif chemokine receptor 1 (CCR1); cytokine-cytokine receptor interaction, including $\mathrm{RAC1}$, signal transducer and activator of transcription (STAT)3 and G protein subunit gamma 10 (GNG10), the hsa04062:chemokine signaling pathway, including CCR1, CCR10, and RAC1; pathways in cancer, including colony stimulating factor 3 receptor (CSF3R); and leukocyte transendothelial migration, including matrix metallopeptidase 9 (MMP9), as presented in Table II. No KEGG pathways were enriched in the downregulated DEGs. Subsequently, the GO term was determined, which included cell-cycle-related downregulated gene alstrom syndrome protein 1 (ALMS1) and inflammatory associated upregulated genes TLR4, CCR1 and CCR10 (Table II).

PPI network construction and module analysis for DEGs. A PPI network was constructed once the DEGs were mapped into the PPI data, including 218 nodes (162 upregulated and 56 downregulated) and 477 edges (Fig. 2). By calculating their degrees, GNG10 (degree, 26), RAC1 (degree, 17), ubiquitin conjugating enzyme E2 D1 (UBE2D1; degree, 16), CCR1 (degree, 12), and CCR10 (degree, 12) were determined to be hub genes (Fig. 3). Furthermore, three significant modules were screened from the PPI network (Fig. 4). Module 1 was involved in ubiquitin-mediated proteolysis, including UBE2D1; module 2 was the chemokine signaling pathway cytokine-cytokine receptor interaction, including CCR1 and CCR10; and module 3 was associated with the cell cycle, including ALMS1 (Table III).

DEGs regulated by miRNA. By searching the mir2disease database, four miRNAs associated with AMI were identified, including hsa-miR-21, hsa-miR-133, hsa-miR-29 and hsa-miR-30c. A total of 1,134 genes were predicted to be target genes of these four miRNAs using the Mirwalk2 database, which were subsequently overlapped with the DEGs. As a result, 48 miRNA-DEG interaction pairs were obtained to construct a miRNA-DEG network that contained three miRNAs (hsa-miR-21-3p, hsa-miR-21-5p and hsa-miR-30c-5p) as well as 29 upregulated and 18 downregulated DEGs (Fig. 5). Examples include MMP9, TLR4, STAT3, CCR1 and ALMS1, which were regulated by hsa-miR-21-5p, whereas RAC1 was one gene regulated by hsa-miR-30c-5p.

The functional enrichment analysis indicated that the DEGs in the network were involved in Hepatitis B (including TLR4 and STAT3), pancreatic cancer (including RAC1 and STAT3) and the proteoglycans associated with cancer (including RAC1, TLR4 and STAT3; Table IV).
Table I. Top 20 upregulated and downregulated differentially expressed genes between acute myocardial infarction patients and healthy controls.

\begin{tabular}{|c|c|c|}
\hline Gene & Log fold change & P-value \\
\hline RNF19B & 1.67 & $1.31 \times 10^{-4}$ \\
\hline TNNT1 & 1.67 & $3.70 \times 10^{-4}$ \\
\hline NFIL3 & 2.30 & $3.91 \times 10^{-4}$ \\
\hline NCF4 & 1.69 & $5.86 \times 10^{-4}$ \\
\hline RNASE2 & 1.29 & $6.91 \times 10^{-4}$ \\
\hline GCA & 1.84 & $7.04 \times 10^{-4}$ \\
\hline CXCL1 & 1.96 & $8.77 \times 10^{-4}$ \\
\hline WBP2 & 1.46 & $9.99 \times 10^{-4}$ \\
\hline STEAP4 & 1.78 & $1.20 \times 10^{-3}$ \\
\hline NINJ1 & 1.37 & $1.46 \times 10^{-3}$ \\
\hline HCAR3 & 3.04 & $1.76 \times 10^{-3}$ \\
\hline S100P & 3.88 & $1.81 \times 10^{-3}$ \\
\hline ATP6V0C & 1.88 & $1.86 \times 10^{-3}$ \\
\hline G6PD & 1.27 & $1.96 \times 10^{-3}$ \\
\hline PTGS2 & 2.44 & $2.07 \times 10^{-3}$ \\
\hline NAMPT & 3.35 & $2.23 \times 10^{-3}$ \\
\hline PLIN3 & 1.20 & $2.27 \times 10^{-3}$ \\
\hline ACSL1 & 2.47 & $2.32 \times 10^{-3}$ \\
\hline TNFAIP6 & 3.23 & $2.35 \times 10^{-3}$ \\
\hline LY96 & 1.54 & $2.72 \times 10^{-3}$ \\
\hline
\end{tabular}

B, Downregulated

\begin{tabular}{lcc}
\hline Gene & Log fold change & P-value \\
\hline RP11-846E15.4 & -1.85 & $6.13 \times 10^{-4}$ \\
MICU3 & -1.62 & $7.23 \times 10^{-4}$ \\
NPTN-IT1 & -1.12 & $1.01 \times 10^{-3}$ \\
FZD6 & -1.37 & $1.27 \times 10^{-3}$ \\
LEF1-AS1 & -1.99 & $1.53 \times 10^{-3}$ \\
PRO0471 & -1.61 & $1.53 \times 10^{-3}$ \\
MAP2K6 & -1.09 & $1.71 \times 10^{-3}$ \\
LOC151657 & -1.33 & $1.79 \times 10^{-3}$ \\
GVINP1 & -1.51 & $1.88 \times 10^{-3}$ \\
CTD-2035E11.5 & -1.02 & $1.91 \times 10^{-3}$ \\
AC007401.2 & -1.09 & $2.13 \times 10^{-3}$ \\
LINC01355 & -1.14 & $2.21 \times 10^{-3}$ \\
C80rf37 & -1.38 & $2.29 \times 10^{-3}$ \\
LOC202181 & -1.67 & $2.30 \times 10^{-3}$ \\
RP11-846E15.2 & -1.72 & $2.58 \times 10^{-3}$ \\
CD3D & -1.10 & $2.61 \times 10^{-3}$ \\
RPS27 & -1.09 & $2.77 \times 10^{-3}$ \\
NDUFA12 & -1.18 & $2.78 \times 10^{-3}$ \\
LOC102724356 & -2.22 & $2.83 \times 10^{-3}$ \\
RPL3 & -1.20 & $2.96 \times 10^{-3}$ \\
\hline
\end{tabular}




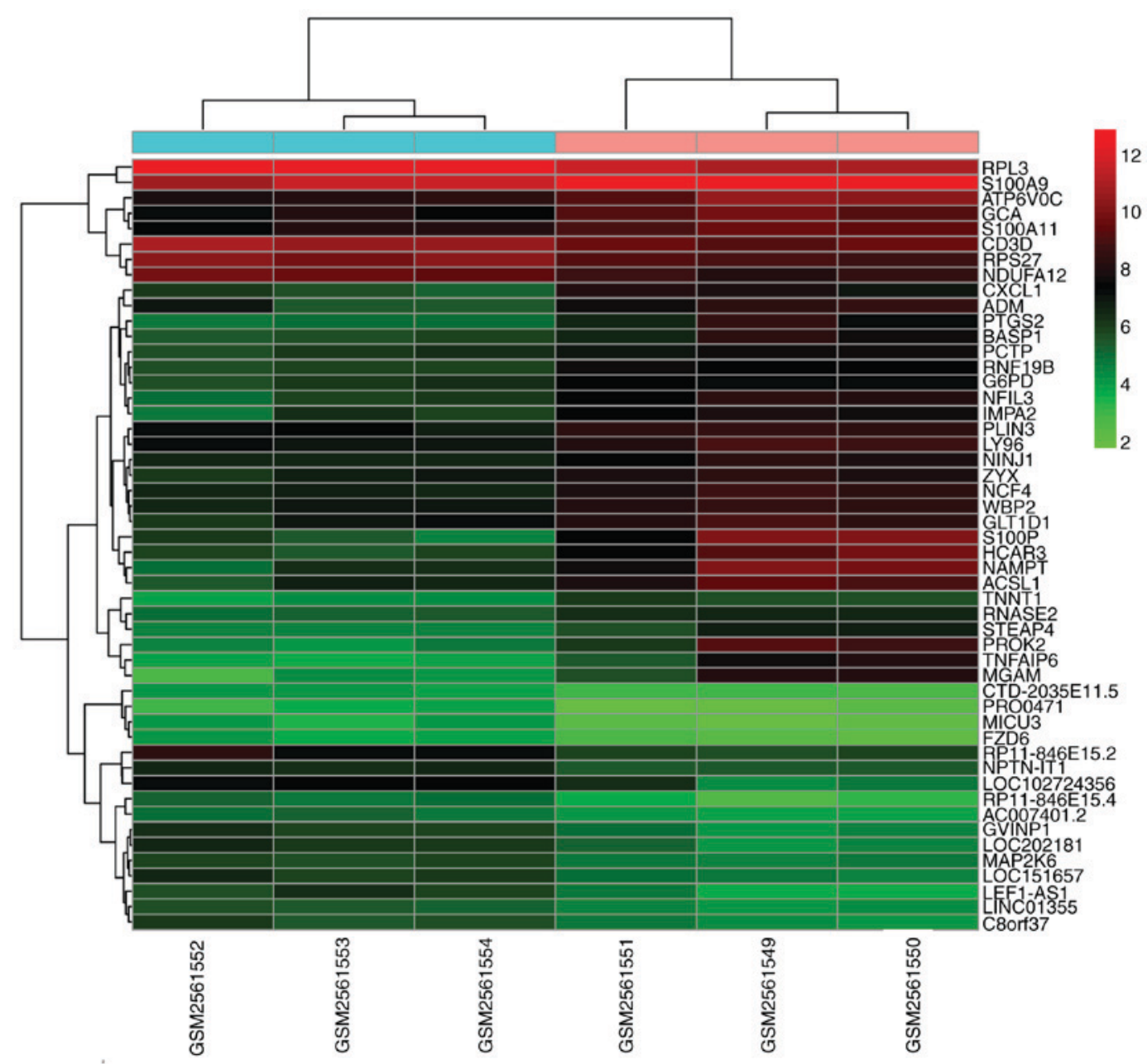

Figure 1. Heat map of the differentially expressed genes among the peripheral blood samples of three patients with acute myocardial infarction and three healthy controls from dataset GSE97320. Expression was presented as the z-score normalized within the samples. The horizontal coordinate represented the sample list. Blue groups represent healthy patients; pink groups represent patients with acute myocardial infarction. Red indicates upregulated expression and green indicates downregulated expression.

Validation analysis. Following data normalization of the GSE24519 dataset, 1,933 genes were identified as DEGs between patients with AMI and controls, including 1,242 upregulated and 691 downregulated genes. For GSE34198, 329 DEGs were screened, consisting of 166 upregulated and 163 downregulated genes; for GSE48060, 1,171 DEGs were obtained, containing 236 upregulated and 935 downregulated genes. A Venn diagram analysis indicated that there were 235 overlapped DEGs between GSE97320 and GSE24519, of which 153 had a consistent expression trend (Fig. 6A). The DEGs of all four datasets were compared, only four common genes were identified, including aldehyde dehydrogenase 8 family member A1 (ALDH8A1), CSF3R, regulator of G protein signaling 10, and CCR10 (Fig. 6B). Among these, the expression levels of ALDH8A1, CSF3R and CCR10 were consistent across the four datasets (Table V).

To investigate the relationship between the 153 DEGs exhibiting a consistent expression trend and clinical characteristics, a subgroup analysis of GSE34198 and GSE48060 was also performed to screen for genes associated with mortality or recurrence. As a result, 1,039 DEGs (864 downregulated and 175 upregulated) were identified between the mortality and non-mortality groups. A total of 1,216 (629 downregulated and 587 upregulated) were screened between the recurrence and non-recurrence groups. Considering the small number of common genes obtained across the four datasets, the shared 153 genes between GSE97320 and GSE24519 were used to overlap with the mortality- or recurrence-associated genes screened in GSE34198 and GSE48060, respectively. Consequently, four (Fig. 6C) and 14 (Fig. 6D) overlaps were separately obtained. However, only three were ultimately suggested as underlying biomarkers for the prediction of mortality [exostosin like glycosyltransferase 2 , chromosome 11 open reading frame 80 , HtrA serine peptidase 1 (HTRA1)] or recurrence (MAP2K2, HTRA1, and MAF1, homolog, negative regulator of RNA polymerase III) in Chinese patients with AMI, due to their consistent expression trends with GSE97320 (Table V).

\section{Discussion}

In the present study, several underlying biomarkers for the diagnosis of AMI in Chinese people were suggested prior to analyses. ALMS1 was demonstrated as a key gene in the PPI module analysis and regulated by miRNAs associated with AMI hsa-miR-21-5p $(25,26)$. In addition, TLR4, MMP9, 


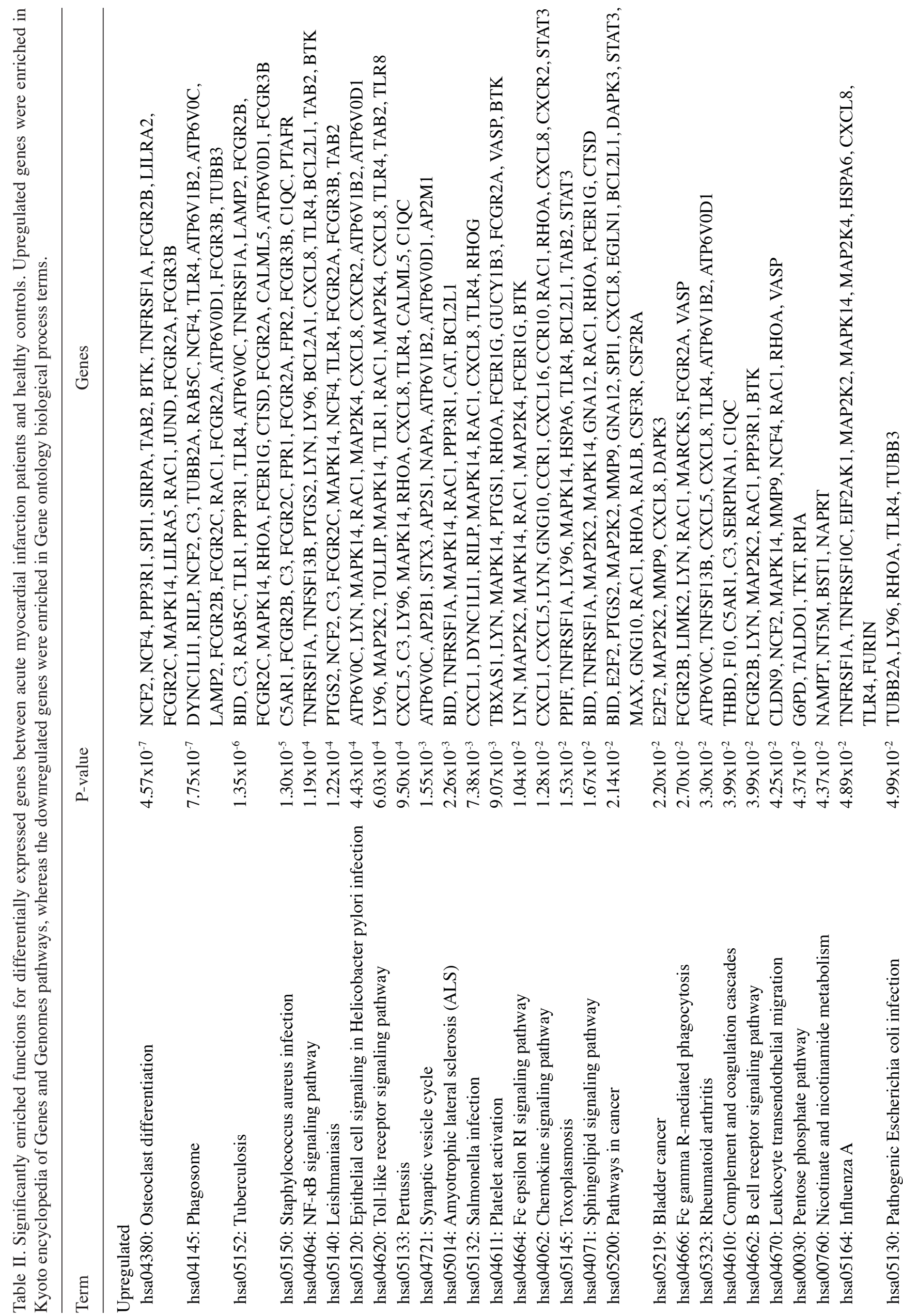




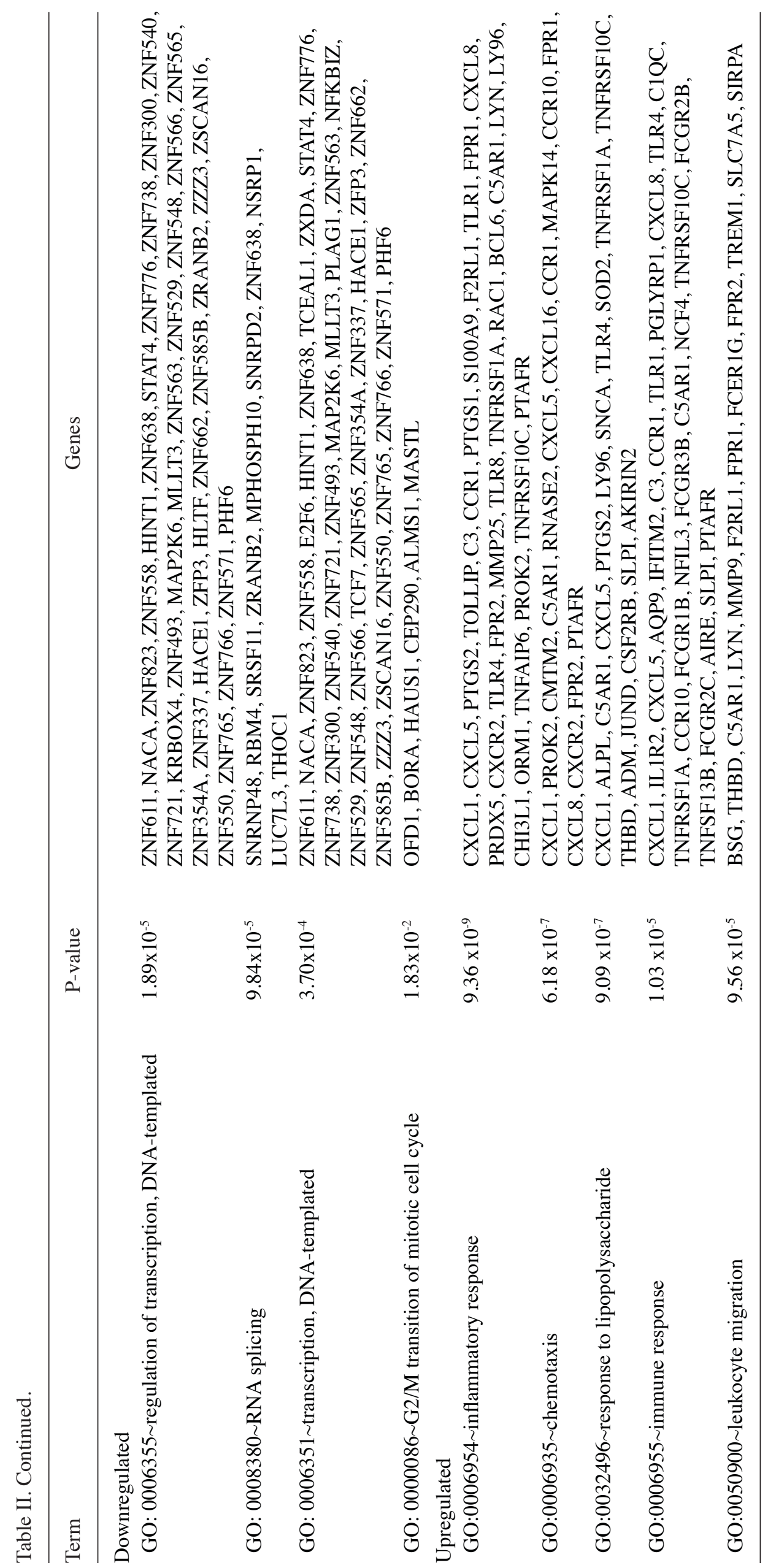




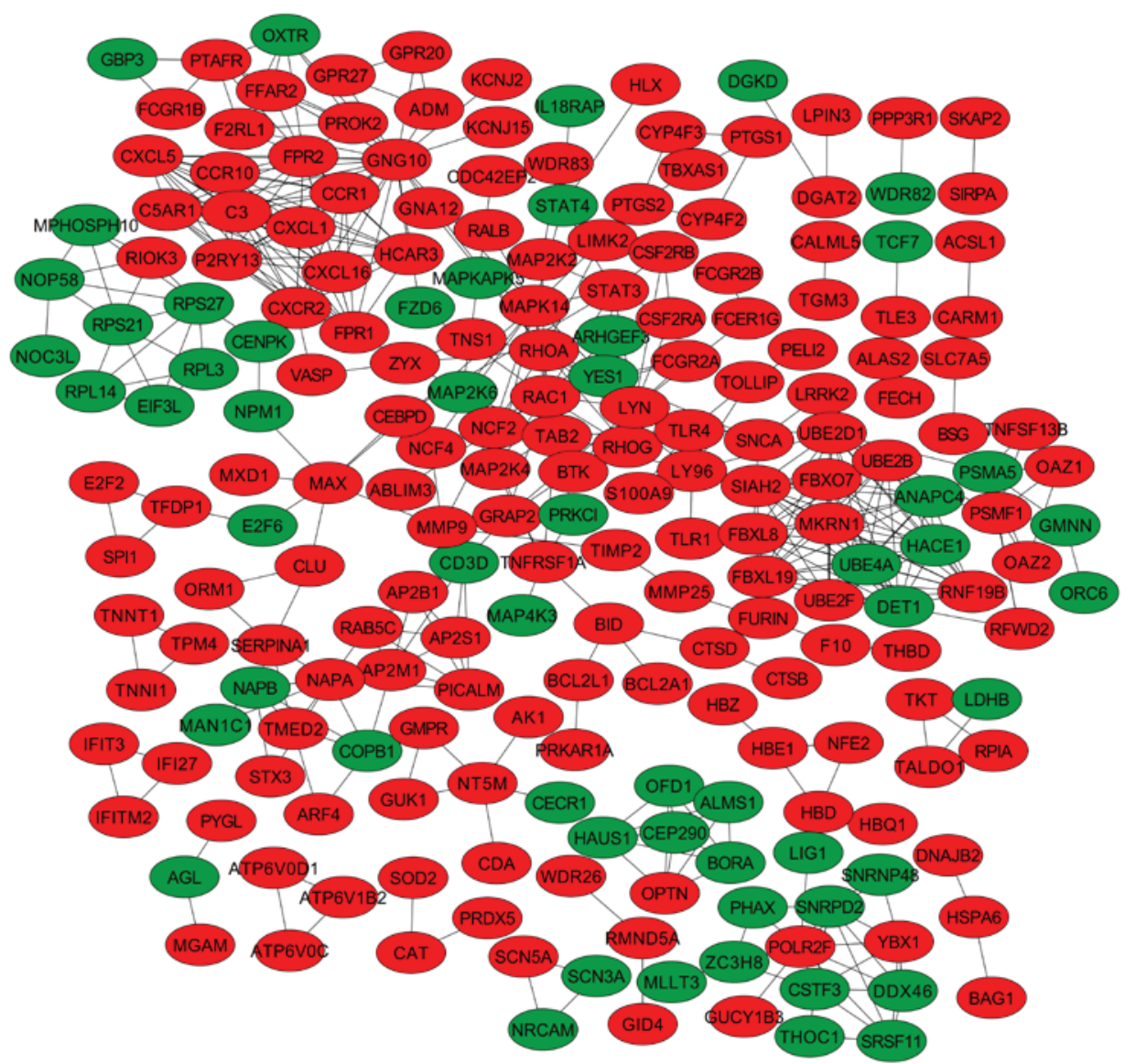

Figure 2. Protein-protein interaction network of differentially expressed genes among the peripheral blood samples of three patients with acute myocardial infarction and three healthy controls. Red indicates the upregulated genes and green indicates the downregulated genes. The network included 218 nodes (162 upregulated and 56 downregulated) and 477 edges.

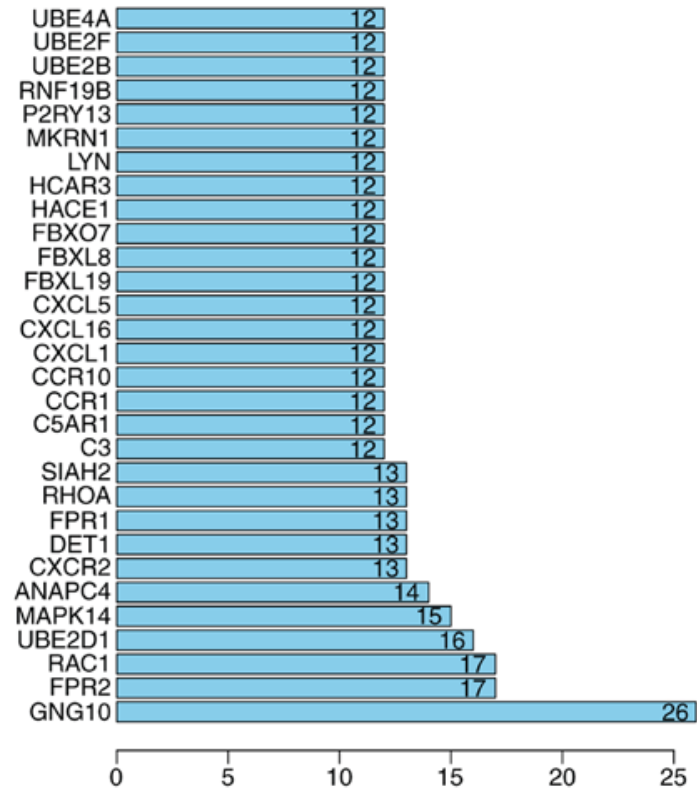

Figure 3. Genes ordered according to the interaction degree in the protein-protein interaction network.

STAT3, CCR1 and RAC1 were likely important, as they are also known to be regulated by miRNAs associated with AMI; hsa-miR-21-5p $(25,26)$ and hsa-miR-30c-5p $(27,28)$. CSF3R and CCR10 were confirmed by comparing Chinese data with international data, and HTRA1 was the only gene associated with both mortality and recurrence.

ALMS1 is responsible for the initiation of Alström syndrome when genetically mutated. Alström syndrome is characterized by retinal degeneration, hearing loss, obesity, diabetes mellitus and cardiomyopathy (29). Additional studies have suggested that ALMS1 functions in the aforementioned diseases by interacting with endosome recycling and/or centrosome-related proteins, including $\alpha$-actinin $1, \alpha$-actinin 4 , myosin $\mathrm{Vb}$, rad50 interacting 1 and huntingtin associated protein $1 \mathrm{~A}$, thereby influencing the cell-cycle pathway (30). ALMS1-deficient fibroblasts proliferate continuously and overexpress extracellular matrix components; this effect likely triggers the excessive remodeling of the normal tissue architecture and results in fibrosis (31). Given that atherosclerosis is associated with the development of intravascular fibrous plaques, ALMS1 mutations may directly contribute to multiorgan fibrotic alterations, ultimately leading to AMI. A glutamic acid repeat polymorphism on exon 1 of the ALMS1 gene is significantly associated with early onset MI (32). Taken together, these studies suggest that ALMS1 is an underlying biomarker for the early diagnosis of AMI. However, no studies have confirmed this hypothesis and additional research is required.

AMI may occur predominantly as a result of atherosclerotic plaque rupture and thrombosis that occludes the 
Table III. Significantly enriched functions for module genes screened from the protein-protein interaction network.

\begin{tabular}{|c|c|c|c|}
\hline Module & Term & P-value & Genes \\
\hline 1 & hsa04120: Ubiquitin mediated proteolysis & $2.85 \times 10^{-9}$ & $\begin{array}{l}\text { UBE4A, ANAPC4, DET1, UBE2F, } \\
\text { UBE2D1, UBE2B }\end{array}$ \\
\hline \multirow[t]{4}{*}{2} & hsa04062: Chemokine signaling pathway & $2.84 \times 10^{-7}$ & $\begin{array}{l}\text { CXCL1, CXCL5, GNG10, CCR1, CXCL16, } \\
\text { CCR10, CXCR2 }\end{array}$ \\
\hline & hsa05150: Staphylococcus aureus infection & $9.44 \times 10^{-5}$ & C5AR1, C3, FPR1, FPR2 \\
\hline & hsa04060: Cytokine-cytokine receptor interaction & $6.401 \times 10^{-3}$ & CCR1, CXCL16, CCR10, CXCR2 \\
\hline & hsa04080: Neuroactive ligand-receptor interaction & $1.07 \times 10^{-2}$ & P2RY13, C5AR1, FPR1, FPR2 \\
\hline \multirow[t]{4}{*}{3} & GO: $0000086 \sim \mathrm{G} 2 / \mathrm{M}$ transition of mitotic cell cycle & $3.36 \times 10^{-11}$ & $\begin{array}{l}\text { OFD1, BORA, HAUS1, CEP290, ALMS1, } \\
\text { OPTN }\end{array}$ \\
\hline & GO: 0007067 mitotic nuclear division & $2.11 \times 10^{-3}$ & OFD1, BORA, HAUS1 \\
\hline & GO: 0042384 cilium assembly & $3.64 \times 10^{-2}$ & CEP290, ALMS1 \\
\hline & GO: 0060271 cilium morphogenesis & $3.98 \times 10^{-2}$ & OFD1, CEP290 \\
\hline
\end{tabular}

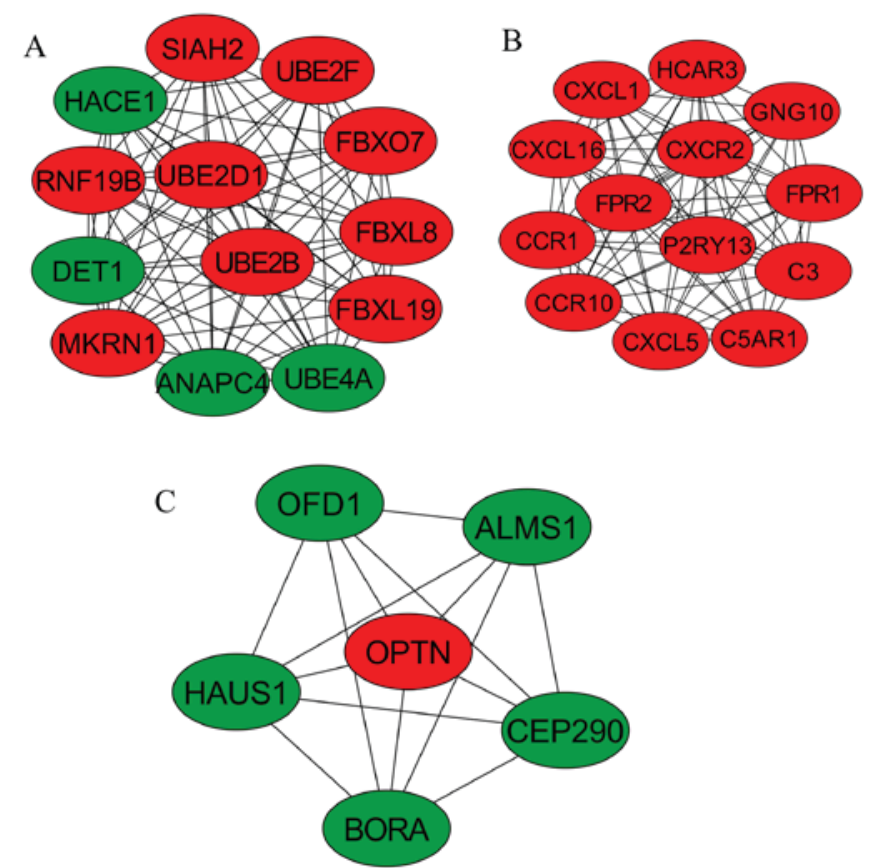

Figure 4. Modules obtained from protein-protein interaction network of differentially expressed genes among the peripheral blood samples of three patients with acute myocardial infarction and three healthy controls. (A) Module 1, (B) module 2 and (C) module 3. Red indicates the upregulated genes and green indicates the downregulated genes.

vessel lumen and significantly lowers the supply of oxygen and metabolites to the myocardium, ultimately inducing cardiomyocyte death $(33,34)$. Inflammation due to bacterial infection (35) or conditions including hypercholesterolemia or hyperlipidemia, are important contributors to atherosclerotic plaque rupture, including both local and systemic inflammation (36). Ishikawa et al (37) performed immunostaining analysis and demonstrated that TLR4 was positively expressed in infiltrated macrophages in ruptured plaque material. In addition, local and systemic levels of TLR4 and TLR2 were significantly higher in patients with AMI, particularly those with cardiovascular events, compared with

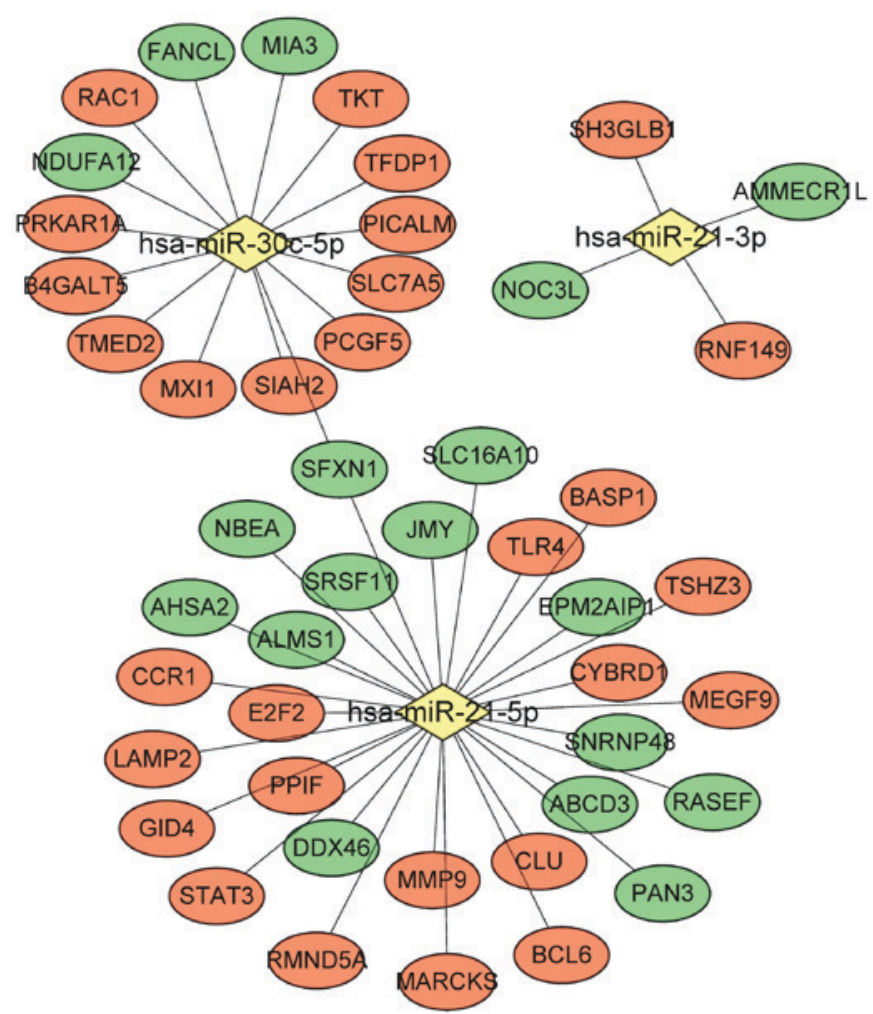

Figure 5. miRNA-gene interaction network. Orange indicates the upregulated genes and green indicates the downregulated genes. All miRNAs were associated with acute myocardial infarction. miRNA/miR, microRNA.

patients with stable angina or controls $(37,38)$. Activated TLRs may weaken the fibrous cap and predispose the plaque to rupture by mediating the high expression of inflammatory genes, including interleukin (IL)-18 receptor (R)1, IL-18R2, IL-8, CCR1, CCR10 and MMP9, via the myeloid differentiation primary response protein MyD88/RAC1/NF- $\kappa \mathrm{B}$ pathway (39-42). Thus, TLRs and their downstream genes may be underlying biomarkers for the early diagnosis of AMI. This theory is supported by the current results and those of a previous study, that revealed the extent of TLR 
Table IV. Significantly enriched pathways for genes in the microRNA-target gene network.

\begin{tabular}{lll}
\hline Term & P-value & Genes \\
\hline hsa05161: Hepatitis B & $1.18 \times 10^{-2}$ & E2F2, MMP9, TLR4, STAT3 \\
hsa05212: Pancreatic cancer & $1.94 \times 10^{-2}$ & E2F2, RAC1, STAT3 \\
hsa05205: Proteoglycans in cancer & $2.76 \times 10^{-2}$ & MMP9, RAC1, TLR4, STAT3
\end{tabular}

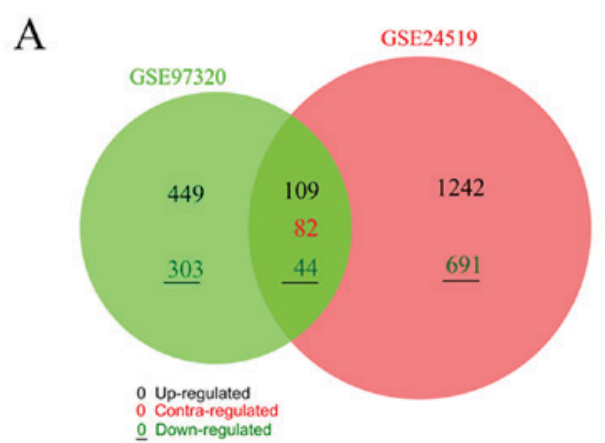

$\mathrm{C}$

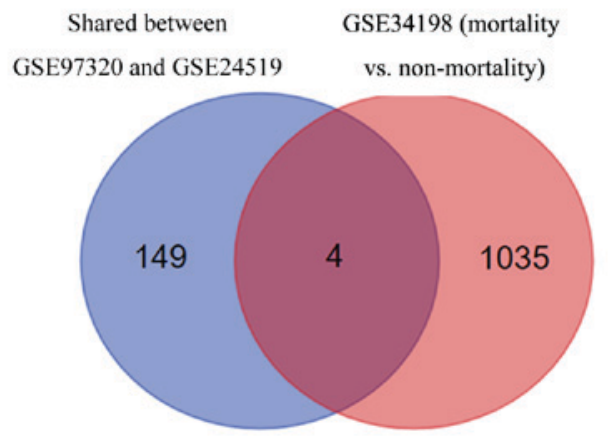

B

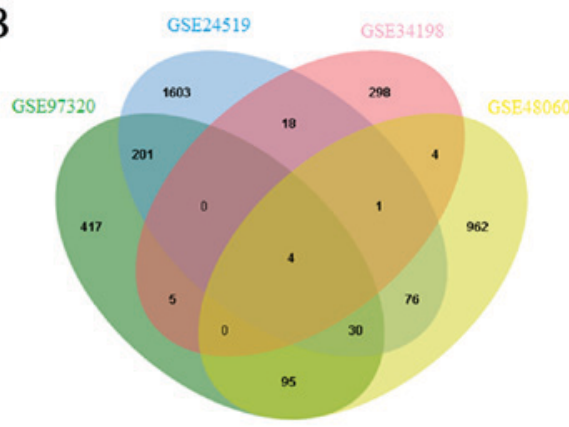

$\mathrm{D}$

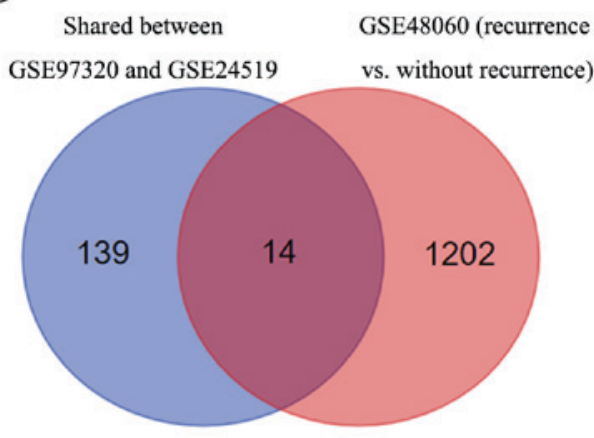

Figure 6. Venn diagram analyses of the common differentially expressed genes among the different datasets. Overlap between differentially expressed genes in (A) GSE97320 and GSE24519 datasets; (B) GSE97320, GSE24519, GSE34198 and GSE48060 datasets; (C) GSE97320 and GSE24519, and GSE34198 datasets; (D) and GSE97320 and GSE24519, and GSE48060 datasets.

activation and IL-18R1/2 expression in circulating cells preceding the excessive release of troponin-T (39), which indicates a potential advantage for their use as biomarkers for early diagnosis compared with troponin-T. Furthermore, high serum MMP-9 levels are associated with an increased risk of AMI (odds ratio, 1.06; 95\% CI, 0.87-1.28) (43), and significantly higher levels of CCR1 are detected in patients with AMI compared with controls (3.76 \pm 0.85 vs. $0.66 \pm 0.19 \mathrm{ng} / \mathrm{ml}, \mathrm{P}<0.05)(44)$. In addition to TLR, the activation of the tyrosine-protein kinase JAK1 (JAK)/STAT signaling pathway has an important role in the onset of AMI by transducing the intracellular signals of various cytokines, such as tumor necrosis factor- $\alpha(\mathrm{TNF}-\alpha)$ (45). Inhibition of the JAK/STAT signaling pathway by AG490 inhibits $\mathrm{NF}-\kappa \mathrm{B}$ protein expression in cardiomyocytes and reduces plasma TNF- $\alpha$ concentrations to prevent the development of AMI (45). However, no studies have directly investigated the diagnostic efficiency of STAT3 in patients with AMI, which may be a future research direction.

Granulocyte colony-stimulating factor (G-CSF) is a multifunctional cytokine that interacts with its receptor G-CSFR, which is encoded by CSF3R. G-CSF promotes inflammation by enhancing the function of mature granulocytes and increasing the number of neutrophils (46). These effects suggest that the expression levels of G-CSF and CSF3R increase with disease progression. This hypothesis was supported by the current study and Li et al (47), who demonstrated that G-CSF and its receptor CSF3R were significantly upregulated in patients with AMI compared with controls and patients with stable angina. However, other studies have suggested that G-CSF and CSF3R improve cardiac function and reduce mortality following AMI by inducing the regeneration of cardiac myocytes and blood vessels through the mobilization of bone marrow stem cells (48), which suggests that high CSF3R expression is a protective response.

HTRA1 is also a gene associated with to oxidative stress and the inflammation response. HTRA1 is highly expressed in activated macrophages and interacts with growth differentiation factor 15 to mediate inflammation and promote cell senescence through the p38 mitogen-activated protein kinase pathway, ultimately predisposing the individual to high risk for the development of age-related macular degeneration $(49,50)$. AMI is particularly prevalent in elderly people (51). Thus, HTRA1 may be important with regard to AMI. In line with 
Table V. Shared differentially expressed genes among different datasets.

A, Differentially expressed genes screened in four datasets

\begin{tabular}{|c|c|c|c|c|c|c|c|c|}
\hline \multirow[b]{2}{*}{ Gene } & \multicolumn{2}{|c|}{ GSE97320 } & \multicolumn{2}{|c|}{ GSE24519a } & \multicolumn{2}{|c|}{ GSE34198 ${ }^{\mathrm{a}}$} & \multicolumn{2}{|c|}{ GSE48060a } \\
\hline & $\log \mathrm{FC}$ & P-value & $\log F C$ & P-value & $\log \mathrm{FC}$ & P-value & $\log F C$ & P-value \\
\hline ALDH8A1 & -1.60 & $1.20 \times 10^{-2}$ & -2.67 & $2.94 \times 10^{-4}$ & -1.94 & $1.03 \times 10^{-2}$ & -1.97 & $7.88 \times 10^{-3}$ \\
\hline CSF3R & 1.06 & $1.22 \times 10^{-2}$ & 1.11 & $3.38 \times 10^{-3}$ & 1.59 & $1.85 \times 10^{-2}$ & 1.20 & $1.6 \times 10^{-4}$ \\
\hline CCR 10 & 1.58 & $8.81 \times 10^{-3}$ & 1.53 & $7.24 \times 10^{-3}$ & 1.18 & $3.86 \times 10^{-2}$ & 1.95 & $2.4 \times 10^{-2}$ \\
\hline
\end{tabular}

B, Mortality-related differentially expressed genes

\begin{tabular}{|c|c|c|c|c|c|c|}
\hline \multirow[b]{2}{*}{ Gene } & \multicolumn{2}{|c|}{ GSE97320a } & \multicolumn{2}{|c|}{ GSE24519a } & \multicolumn{2}{|c|}{ GSE34198b } \\
\hline & $\log \mathrm{FC}$ & P-value & $\log \mathrm{FC}$ & P-value & $\log \mathrm{FC}$ & P-value \\
\hline EXTL2 & -2.17 & $3.54 \times 10^{-2}$ & -1.01 & $8.24 \times 10^{-3}$ & -1.70 & $2.75 \times 10^{-3}$ \\
\hline C11orf80 & -1.35 & $1.50 \times 10^{-2}$ & -1.85 & $2.92 \times 10^{-2}$ & -1.98 & $2.31 \times 10^{-4}$ \\
\hline HTRA1 & 1.06 & $1.18 \times 10^{-2}$ & 1.11 & $6.42 \times 10^{-3}$ & 2.56 & $1.09 \times 10^{-2}$ \\
\hline
\end{tabular}

C, Recurrence-related differentially expressed genes

\begin{tabular}{|c|c|c|c|c|c|c|}
\hline \multirow[b]{2}{*}{ Gene } & \multicolumn{2}{|c|}{ GSE97320a } & \multicolumn{2}{|c|}{ GSE24519ª } & \multicolumn{2}{|c|}{ GSE48060 } \\
\hline & $\log \mathrm{FC}$ & $\mathrm{P}$-value & $\log \mathrm{FC}$ & P-value & $\log F C$ & P-value \\
\hline MAP2K2 & 1.17 & $1.74 \times 10^{-2}$ & 1.23 & $3.46 \times 10^{-2}$ & 1.10 & $2.65 \times 10^{-2}$ \\
\hline HTRA1 & 1.06 & $1.18 \times 10^{-2}$ & 1.11 & $6.42 \times 10^{-3}$ & 1.81 & $1.36 \times 10^{-2}$ \\
\hline MAF1 & 1.05 & $6.78 \times 10^{-3}$ & 1.10 & $9.42 \times 10^{-3}$ & 1.84 & $1.42 \times 10^{-2}$ \\
\hline
\end{tabular}

FC, fold-change. ${ }^{a}$ AMI vs. control; ${ }^{b}$ mortality vs. non-mortality; crecurrence vs. non-recurrence.

the above study, the present study demonstrated that HTRA1 was significantly upregulated in the AMI group, particularly in patients who succumbed to the disease following a recurrence.

In conclusion, the present study preliminarily suggests that inflammation markers, including STAT3, CCR1, RAC1, MMP9, CCR10, CSF3R and HTRA1, as well as cell-cycle-associated genes including ALMS1, have a potential role that is crucial for the diagnosis and prognosis of AMI in Chinese people. However, additional clinical studies are required to confirm the above findings, which represents a limitation of the present study.

\section{Acknowledgements}

Not applicable.

\section{Funding}

No funding was received.

\section{Availability of data and materials}

The datasets generated and/oranalyzed during the current study areavailable in the NCBIdatabase repository [https://www.ncbi.
nlm.nih.gov/geo/query/acc.cgi?acc=GSE97320; https://www. ncbi.nlm.nih.gov/geo/query/acc.cgi?acc=GSE24519; https://www.ncbi. nlm.nih.gov/geo/query/acc.cgi?acc=GSE34198; https://www. ncbi.nlm.nih.gov/geo/query/acc.cgi?acc=GSE48060].

\section{Author's contributions}

JS and CG participated in the design of this study. JS and CG performed the statistical and bioinformatics analyses. RW, CX and MY contributed to the acquisition and interpretation of data. JS and CG drafted and revised the manuscript. All authors read and approved the final manuscript.

\section{Ethics approval and consent to participate}

Not applicable.

\section{Consent for publication}

Not applicable.

\section{Competing interests}

The authors declare that they have no competing interests. 


\section{References}

1. Wang DZ, Shen CF, Zhang Y, Zhang H, Song GD, Li W, Xue XD, $\mathrm{Xu}$ ZL, Zhang S and Jiang GH: Fifteen-year trend in incidence of acute myocardial infarction in Tianjin of China. Zhonghua Xin Xue Guan Bing Za Zhi 45: 154-159, 2017 (In Chinese).

2. Chang J, Liu X and Sun Y: Mortality due to acute myocardial infarction in China from 1987 to 2014: Secular trends and age-period-cohort effects. Int J Cardiol 227: 229-238, 2017.

3. Chen Y, Jiang L, Smith M, Pan H, Collins R, Peto R and Chen Z; COMMIT/CCS-2 collaborative group: Sex differences in hospital mortality following acute myocardial infarction in China: Findings from a study of 45852 patients in the COMMIT/CCS-2 study. Heart Asia 3: 104-110, 2011.

4. Reichlin T, Irfan A, Twerenbold R, Reiter M, Hochholzer W, Burkhalter H, Bassetti S, Steuer S, Winkler K, Peter F, et al: Utility of absolute and relative changes in cardiac troponin concentrations in the early diagnosis of acute myocardial infarction. Circulation 124: 136-145, 2011.

5. Rubini Gimenez M, Twerenbold R, Jaeger C, Schindler C, Puelacher C, Wildi K, Reichlin T, Haaf P, Merk S, Honegger $\mathrm{U}$, et al: One-hour rule-in and rule-out of acute myocardial infarction using high-sensitivity cardiac troponin I. Am J Med 128: 861-870.e4, 2015.

6. McCann CJ, Glover BM, Menown IB, Moore MJ, McEneny J, Owens CG, Smith B, Sharpe PC, Young IS and Adgey JA: Novel biomarkers in early diagnosis of acute myocardial infarction compared with cardiac troponin T. Eur Heart J 29: 2843-2850, 2008.

7. Maznyczka A, Kaier T and Marber M: Troponins and other biomarkers in the early diagnosis of acute myocardial infarction. Postgrad Med J 91: 322-330, 2015.

8. Roongsritong C, Warraich I and Bradley C: Common causes of troponin elevations in the absence of acute myocardial infarction: Incidence and clinical significance. Chest 125: 1877-1884, 2004.

9. Mcmahon CG, Lamont JV, Curtin E, McConnell RI, Crockard M, Kurth MJ, Crean P and Fitzgerald SP: Diagnostic accuracy of heart-type fatty acid-binding protein for the early diagnosis of acute myocardial infarction. Am J Emerg Med 30: 267-274, 2012

10. Pyati AK, Devaranavadagi BB, Sajjannar SL, Nikam SV, Shannawaz M and Sudharani: Heart-type fatty acid binding protein: A better cardiac biomarker than CK-MB and myoglobin in the early diagnosis of acute myocardial infarction. J Clin Diagn Res 9: BC08-BC11, 2015.

11. Wang J, Tang B, Liu X, Wu X, Wang H, Xu D and Guo Y: Increased monomeric CRP levels in acute myocardial infarction: A possible new and specific biomarker for diagnosis and severity assessment of disease. Atherosclerosis 239: 343-349, 2015.

12. Han QY, Wang HX, Liu XH, Guo CX, Hua Q, Yu XH, Li N, Yang YZ, Du J, Xia YL and Li HH: Circulating E3 ligases are novel and sensitive biomarkers for diagnosis of acute myocardial infarction. Clin Sci (Lond) 128: 751-760, 2015.

13. Irizarry RA, Hobbs B, Collin F, Beazer-Barclay YD, Antonellis KJ, Scherf U and Speed TP: Exploration, normalization, and summaries of high density oligonucleotide array probe level data. Biostatistics 4: 249-264, 2003.

14. R Development Core Team: R: A language and environment for statistical computing. The R Foundation for Statistical Computing, Vienna, 2009.

15. Ritchie ME, Phipson B, Wu D, Hu Y, Law CW, Shi W and Smyth GK: limma powers differential expression analyses for RNA-sequencing and microarray studies. Nucleic Acids Res 43: e47, 2015 .

16. Szklarczyk D, Franceschini A, Wyder S, Forslund K, Heller D, Huerta-Cepas J, Simonovic M, Roth A, Santos A, Tsafou KP, et al: STRING v10: Protein-protein interaction networks, integrated over the tree of life. Nucleic Acids Res 43 (Database Issue) D447-D452, 2015

17. Kohl M, Wiese S and Warscheid B: Cytoscape: Software for visualization and analysis of biological networks. Methods Mol Biol 696: 291-303, 2011

18. Bader GD and Hogue CW: An automated method for finding molecular complexes in large protein interaction networks. BMC Bioinformatics 4: 2, 2003.

19. Jiang Q, Wang Y, Hao Y, Juan L, Teng M, Zhang X, Li M, Wang $\mathrm{G}$ and Liu Y: miR2disease: A manually curated database for microRNA deregulation in human disease. Nucleic Acids Res 37 (Database Issue): D98-D104, 2009.
20. Dweep H and Gretz N: miRWalk2.0: A comprehensive atlas of microRNA-target interactions. Nat Methods 12: 697, 2015.

21. Ogata H, Goto S, Sato K, Fujibuchi W, Bono H and Kanehisa M: KEGG: Kyoto encyclopedia of genes and genomes. Nucleic Acids Res 27: 29-34, 1999.

22. Ashburner M, Ball CA, Blake JA, Botstein D, Butler H, Cherry JM, Davis AP, Dolinski K, Dwight SS, Eppig JT, et al: Gene ontology: Tool for the unification of biology. The gene ontology consortium. Nat Genet 25: 25-29, 2000.

23. Zdeněk V, Ivan M, M. Feglarová K, Peleška P, Marie JT, Jan-Slovák K and Jana DZ: Determinants of excess genetic risk of acute myocardial infarction-a matched case-control study. Eur J Biomed Informatics 8: 34-43, 2012.

24. Suresh R, Li X, Chiriac A, Goel K, Terzic A, Perez-Terzic C and Nelson TJ: Transcriptome from circulating cells suggests dysregulated pathways associated with long-term recurrent events following first-time myocardial infarction. J Mol Cell Cardiol 74: 13-21, 2014

25. Wang F, Long G, Zhao C, Li H, Chaugai S, Wang Y, Chen C and Wang DW: Atherosclerosis-related circulating miRNAs as novel and sensitive predictors for acute myocardial infarction. PLoS One 9: e105734, 2014

26. Zhang Y, Liu YJ, Liu T, Zhang $\mathrm{H}$ and Yang SJ: Plasma microRNA-21 is a potential diagnostic biomarker of acute myocardial infarction. Eur Rev Med Pharmacol Sci 20: 323-329, 2016.

27. Huang Y, Chen J, Zhou Y, Yu X, Huang C, Li J and Feng Y: Circulating miR-30 is related to carotid artery atherosclerosis. Clin Exp Hypertens 38: 489-494, 2016.

28. Irani S, Pan X, Peck BC, Iqbal J, Sethupathy P and Hussain MM: MicroRNA-30c mimic mitigates hypercholesterolemia and atherosclerosis in mice. J Biol Chem 291: 18397-18409, 2016.

29. Collin GB, Marshall JD, Ikeda A, So WV, Russell-Eggitt I, Maffei P, Beck S, Boerkoel CF, Sicolo N, Martin M, et al: Mutations in ALMS1 cause obesity, type 2 diabetes and neurosensory degeneration in Alström syndrome. Nat Genet 31: 74-78, 2002.

30. Collin GB, Marshall JD, King BL, Milan G, Maffei P, Jagger DJ and Naggert JK: The Alström syndrome protein, ALMS1, interacts with $\alpha$-actinin and components of the endosome recycling pathway. PLoS One 7: e37925, 2012.

31. Zulato E, Favaretto F, Veronese C, Campanaro S, Marshall JD, Romano S, Cabrelle A, Collin GB, Zavan B, Belloni AS, et al: ALMS1-deficient fibroblasts over-express extra-cellular matrix components, display cell cycle delay and are resistant to apoptosis. PLoS One 6: e19081, 2011.

32. Ichihara S, Yamamoto K, Asano H, Nakatochi M, Sukegawa M, Ichihara G, Izawa H, Hirashiki A, Takatsu F, Umeda H, et al: Identification of a glutamic acid repeat polymorphism of ALMS1 as a novel genetic risk marker for early-onset myocardial infarction by genome-wide linkage analysis. Circ Cardiovasc Genet 6: $569-578,2013$.

33. Ojio S, Takatsu H, Tanaka T, Ueno K, Yokoya K, Matsubara T, Suzuki T, Watanabe S, Morita N, Kawasaki M, et al: Considerable time from the onset of plaque rupture and/or thrombi until the onset of acute myocardial infarction in humans: Coronary angiographic findings within 1 week before the onset of infarction. Circulation 102: 2063-2069, 2000.

34. Park JS, Shin DG, Kim YJ, Hong GR and Cho IH: Acute myocardial infarction as a consequence of stent fracture and plaque rupture after sirolimus-eluting stent implantation. Int J Cardiol 134: e79-e81, 2009.

35. Ohki T, Itabashi Y, Kohno T, Yoshizawa A, Nishikubo S, Watanabe S, Yamane G and Ishihara K: Detection of periodontal bacteria in thrombi of patients with acute myocardial infarction by polymerase chain reaction. Am Heart J 163: 164-167, 2012.

36. Tanaka A, Shimada K, Sano T, Namba M, Sakamoto T, Nishida Y, Kawarabayashi T, Fukuda D and Yoshikawa J: Multiple plaque rupture and $\mathrm{C}$-reactive protein in acute myocardial infarction. J Am Coll Cardiol 45: 1594-1599, 2005.

37. Ishikawa Y, Satoh M, Itoh T, Minami Y, Takahashi Y and Akamura M: Local expression of Toll-like receptor 4 at the site of ruptured plaques in patients with acute myocardial infarction. Clin Sci (Lond) 115: 133-140, 2008.

38. Satoh S, Yada R, Inoue H, Omura S, Ejima E, Mori T, Takenaka K, Kawamura N, Numaguchi K, Mori E, et al: Toll-like receptor-4 is upregulated in plaque debris of patients with acute coronary syndrome more than Toll-like receptor-2. Heart Vessels 31: 1-5, 2016. 
39. van der Pouw Kraan TC, Bernink FJ, Yildirim C, Koolwijk P, Baggen JM, Timmers L, Beek AM, Diamant M, Chen WJ, van Rossum AC, et al: Systemic toll-like receptor and interleukin-18 pathway activation in patients with acute ST elevation myocardial infarction. J Mol Cell Cardiol 67: 94-102, 2014.

40. Yang Y, Lv J, Jiang S, Ma Z, Wang D, Hu W, Deng C, Fan C, Di S, Sun Y and Yi W: The emerging role of Toll-like receptor 4 in myocardial inflammation. Cell Death Dis 7: e2234, 2016.

41. Arbibe L, Mira JP, Teusch N, Kline L, Guha M, Mackman N, Godowski PJ, Ulevitch RJ and Knaus UG: Toll-like receptor 2-mediated NF-kappa B activation requires a Rac1-dependent pathway. Nat Immunol 1: 533-540, 2000.

42. Nie W, Lv Y, Yan L, Guan T, Li Q, Guo X, Liu W, Feng M, Xu G, Chen X and Lv H: Discovery and characterization of functional modules and pathogenic genes associated with the risk of coronary artery disease. Rsc Adv 5: 26443-26451, 2015.

43. Buduneli E, Mäntylä P, Emingil G, Tervahartiala T, Pussinen P, Barıs N, Akıllı A, Atilla G and Sorsa T: Acute myocardial infarction is reflected in salivary matrix metalloproteinase- 8 activation level. J Periodontol 82: 716-725, 2011.

44. Kang B and Mao Y: Detection of bilirubin, CCR1, troponin I and IL-6 in patients with acute myocardial infarction. Xi Bao Yu Fen Zi Mian Yi Xue Za Zhi 30: 1198-1200, 2014 (In Chinese).

45. Zhang S, Liu X, Goldstein S, Li Y, Ge J, He B, Fei X, Wang Z and Ruiz G: Role of the JAK/STAT signaling pathway in the pathogenesis of acute myocardial infarction in rats and its effect on NF- $\kappa$ B expression. Mol Med Rep 7: 93-98, 2013.
46. Molineux G: Granulocyte colony-stimulating factors. Cancer Treat Res 157: 33-53, 2011.

47. Li CR, Wang LM, Gong Z, Jiang JF, Duan QL, Yan WW and Liu XH: Expression characteristics of neutrophil and mononuclear-phagocyte related genes mRNA in the stable angina pectoris and acute myocardial infarction stages of coronary artery disease. J Geriatr Cardiol 12: 279-286, 2015.

48. Takano H, Ohtsuka M, Akazawa H, Toko H, Harada M, Hasegawa H, Nagai T and Komuro I: Pleiotropic effects of cytokines on acute myocardial infarction: G-CSF as a novel therapy for acute myocardial infarction. Curr Pharm Des 9: 1121-1127, 2003.

49. Supanji, Shimomachi M, Hasan MZ, Kawaichi M and Oka C: HtrAl is induced by oxidative stress and enhances cell senescence through p38 MAPK pathway. Exp Eye Res 112: 79-92, 2013.

50. Stanton C, Kortvely E, Hayward C and Ueffing M; Alan wright: The serine protease HTRA1 is a potential regulator of the inflammatory cytokine GDF15. Invest Ophthalmol Vis Sci 54: 5006, 2013.

51. Damiani G, Salvatori E, Silvestrini G, Ivanova I, Bojovic L, Iodice L and Ricciardi W: Influence of socioeconomic factors on hospital readmissions for heart failure and acute myocardial infarction in patients 65 years and older: Evidence from a systematic review. Clin Interv Aging 10: 237-245, 2015.

This work is licensed under a Creative Commons

Attribution-NonCommercial-NoDerivatives 4.0 International (CC BY-NC-ND 4.0) License. 